\title{
Perioperative Outcomes of Open Radical Prostatectomy versus Laparoscopic Radical Prostatectomy in Asian Men: Comparison of Two Initial Series by the same Surgeon
}

\author{
Sunai Leewansangtong, Wirat Wiangsakunna, Tawatchai Taweemankongsap
}

Division of Urology, Department of Surgery, Faculty of Medicine Siriraj Hospital, Mahidol University, Bangkoknoi, Bangkok, Thailand

\begin{abstract}
Purpose: To compare the perioperative outcomes in 2 initial series of open radical prostatectomy (ORP) and laparoscopic radical prostatectomy (LRP) in Asian men with prostate cancer.

Materials and Methods: From March 1999 to February 2007, the first 100 consecutive patients who underwent ORP and the first 100 consecutive patients who underwent LRP by the same surgeon (SL) were assessed. Mean age, clinical stage, preoperative PSA level, Gleason score, operative time, estimated blood loss, blood transfusion rate, perioperative complications, pathological stage and margin status were compared between the 2 groups.

Results: For each 100 patients in ORP and LRP, mean age and clinical stage were not significantly different. The operative time in LRP was significantly longer than ORP (188 \pm 55 versus $114 \pm 31$ minute, $\mathrm{p}$ value $=0.01)$. Mean estimated blood loss and blood transfusion rate in LRP was significantly lower than ORP, $521 \pm 328$ versus $809 \pm 510 \mathrm{~mL}(\mathrm{p}$ value $=0.03$ ) and $27 \%$ versus $55 \%(\mathrm{p}$ value $=0.01)$, respectively. For pathological organ confined disease, the free surgical margin rate of ORP and LRP was not significantly different $(88.9 \%$ versus $91.3 \%$, respectively, $\mathrm{p}=0.58)$. There was no significant major complication in either group.

Conclusions: For initial experience by a single surgeon, LRP is comparable to ORP with no significant morbidity. LRP had longer operative time. However, LRP decreased blood loss and blood transfusion. For localized prostate cancer, free surgical margin rate of ORP and LRP was not significantly different.
\end{abstract}

Key words: prostate neoplasms; prostatectomy; laparoscopy

Int Braz, J Urol. 2009; 35: 151-7

\section{INTRODUCTION}

Radical prostatectomy has been a standard treatment for clinical localized prostate cancer. Open retropubic radical prostatectomy (ORP) has been accepted as a standard technique (1-3). For over a decade, minimal invasive surgery techniques have been widely used for prostate cancer in urology, and laparoscopic radical prostatectomy (LRP) in particular has become a standard treatment for clinically localized prostate cancer (4-6). Reported data in western countries have shown that early LRP results could be comparable to ORP in terms of operative parameters, morbidity, urinary function, sexual function and oncological outcome (4,7-9). Furthermore, several studies have shown various advantages of LRP. During LRP the structure of prostate gland, urethra, bladder, neurovascular bundle and other surrounding tissues are magnified. Thus, LRP is associated with less blood loss and blood transfusion. Patients who undergo LRP experience less postoperative pain, require fewer analgesic drugs and reduce their hospital stay. However, 
LRP requires longer operative time and is more costly than ORP. Since there are few published data regarding these issues in South East Asia, this study was conducted in Thai men to compare perioperative data and pathological outcomes between LRP and ORP. To reduce variations in surgical skill and experience of the surgeon, all patients in two initial series of ORP and LRP were operated on by single surgeon.

\section{MATERIALS AND METHODS}

From March 1999 to February 2007, the first 100 consecutive patients who underwent ORP and the first 100 consecutive patients who underwent LRP by single surgeon (SL) at Siriraj Hospital, Bangkok, were retrospectively evaluated. Pathology results in all patients confirmed adenocarcinoma of the prostate gland. All patients had clinical localized (cT1 or cT2) or clinical locally advanced (cT3) prostate cancer and negative bone $\mathrm{CT}$ scan. In each group, most patients were diagnosed using a transrectal ultrasound guide biopsy if they had elevated prostate-specific antigen (PSA) or abnormal digital rectal examination (DRE) or both. However, some patients with previous transurethral prostatectomy (TURP) were diagnosed with prostate cancer somewhere else and referred to our hospital for further definite treatment. All treatment options were informed and decided by the patients alone. During 1999-2004, all patients in this series underwent ORP. LRP in this series was initially performed in February 2005, then, LRP was performed parallel to ORP. In 2006 and 2007, almost all patients in this series underwent LRP. All surgical procedure options were also decided by the patients themselves. All ORPs were performed using the retrograde technique described by Walsh (10). Of the 100 patients with LRP, 42 and 58 patients underwent the procedure via an intraperitoneal or extraperitoneal approach, respectively. To compare the perioperative data, morbidity and pathological outcomes between ORP and LRP, patients' age, clinical stage, preoperative PSA level, Gleason score, operating time, estimated blood loss, blood transfusion rate, perioperative complications, pathological stage and margin status were compared. Mean data were compared using the Student's t-test. Chi-square test was used for descriptive data. All data were analyzed by SPSS software program.

\section{RESULTS}

The patient characteristics of age, clinical stage, preoperative PSA level and Gleason score between ORP and LRP are shown in Table-1. Mean age and clinical stage were not different between ORP and LRP. Mean preoperative PSA level in ORP was higher than that in LRP. However, mean Gleason Score in LRP was higher than that in ORP. Of 100 patients in each group, 8 in ORP and 17 patients in LRP had previous TURP. Table- 2 shows operative and pathological data in both groups. The operative time in LRP was significantly higher than that in ORP. In contrast, estimated blood loss and blood transfusion rate were significantly lower in LRP. Of 100 patients in ORP, 72 and 28 patients had pathological localized (pT2) and pathological locally advanced disease (pT3), respectively. Of 100 patients in LRP, 69 and

Table 1 - Patient characteristics in ORP and LRP.

\begin{tabular}{lccc}
\hline & ORP & LRP & p Value \\
\hline No. of patients & 100 & 100 & \\
Mean age (years) & $68.6(\mathrm{SD}=6.5)$ & $65.5(\mathrm{SD}=7.0)$ & 0.61 \\
Preoperative PSA $(\mathrm{ng} / \mathrm{mL})$ & $18.1(\mathrm{SD}=19.1)$ & $13.8(\mathrm{SD}=12.2)$ & 0.02 \\
Gleason score & $6.5(\mathrm{SD}=1.2)$ & $7.1(\mathrm{SD}=0.8)$ & 0.01 \\
No. clinical stage T1, T2 & 90 & 87 & 0.56 \\
No. clinical stage T3 & 10 & 13 & 0.56 \\
\hline
\end{tabular}

$O R P=$ open retropubic radical prostatectomy; $L R P=$ laparoscopic radical prostatectomy; $S D=$ standard deviation. 
Open RRP versus LRP in Asian Men

Table 2-Operative and pathological data in 100 patients of ORP and 100 patients of LRP.

\begin{tabular}{lccc}
\hline & ORP & LRP & p Value \\
\hline Operative time (min.) & $114(\mathrm{SD}=31)$ & $188(\mathrm{SD}=55)$ & 0.01 \\
Estimate blood loss (mL) & $809(\mathrm{SD}=510)$ & $521(\mathrm{SD}=328)$ & 0.03 \\
Blood transfusion (\%) & 55 & 27 & 0.01 \\
Pathological localized stage $(\%)$ & 72 & 69 & 0.64 \\
Over all free margin rate $(\%)$ & 73 & 71 & 0.38 \\
\hline
\end{tabular}

$O R P=$ open retropubic radical prostatectomy; $L R P=$ laparoscopic radical prostatectomy; $S D=$ standard deviation .

31 patients had pT2 and pT3, respectively. In both groups, there was no significantly different proportion of the prostate affected or a locally advanced disease. Overall free margin rate in ORP and LRP were not significant different $(\mathrm{p}=0.38)$. Table-3 shows margin status in pT2 and pT3 stage between ORP and LRP. Of 72 and 69 patients with pT2 in ORP and LRP, 64 $(88.9 \%)$ and $63(91.3 \%)$ margin free, respectively. It was not significantly different $(\mathrm{P}=0.58)$. In $\mathrm{pT} 3$, positive margin rate were high in both groups. However, it was not significantly different between ORP and LRP $(p=0.43)$. For pathological stage and margin status, our data showed that ORP and LRP were also not statistically different.

There were 3 complications in ORP. One patient had prolonged urinary leakage for 2 weeks and conservatively treated. Two patients had wound infection. For LRP, 5 patients required a conversion to ORP. Three patients had no progression in surgical technique for LRP due to the learning curve.
All of these 3 patients were in the first 5 patients in this series. One patient had $\mathrm{CO}_{2}$ retention during suturing of an anastomosis and needed a conversion to ORP. Another had incidental colonic cancer and also required conversion to colonic resection. There was prolonged urinary leakage in 3 patients in LRP. However, all patients were treated conservatively. One patient in LRP had urine collection in pelvic cavity due to voiding obstruction. There was no rectal injury in either group.

All patients had no preoperative incontinence in either group. After surgery, we defined incontinence status as 3 degrees according to the number of pads used for the entire day. No pad was considered as any incontinence, 1 or 2 pads were considered as incontinence and 3 or more pads were considered as severe incontinence. For 6 months, of 100 patients in ORP, 90 patients had no pad, 7 patients had incontinence that needed 1 or 2 pads. Three patients had severe incontinence that required 3 or more pads or other de-

Table 3 - Surgical margin in pathological localized disease and pathological locally advanced disease between ORP and LRP.

\begin{tabular}{lccc}
\hline & Number of Patients & \\
& ORP & LRP & p Value \\
\hline Localized staging & 72 & 69 & \\
$\quad$ Free margin & $64(88.9 \%)$ & $63(91.3 \%)$ & 0.58 \\
$\quad$ Positive margin & $8(11.1 \%)$ & $6(8.7 \%)$ & 0.58 \\
Locally advanced staging & 28 & 31 & \\
$\quad$ Free margin & $9(32.1 \%)$ & $8(25.8 \%)$ & 0.43 \\
$\quad$ Positive margin & $19(67.9 \%)$ & $23(74.2 \%)$ & 0.43 \\
\hline
\end{tabular}

ORP = open retropubic radical prostatectomy; $L R P=$ laparoscopic radical prostatectomy. 
vices. For 100 patients in LRP, 92 patients had no pad, 6 patients had incontinence that needed 1 or 2 pads. Two patients had severe incontinence that needed 3 or more pads or other devices. As regards potency, it was difficult to evaluate in this series. Bilateral nerve sparing procedure did not performed in the patients with high-risk cancer such as cT3, PSA higher than $10 \mathrm{ng} / \mathrm{mL}$, Gleason score 8 or above, patients who had previous impotence or patients who did not have sexual interest. In addition, end point of this study was short for perioperative period. Thus, our data regarding potency was limited. However, several of the younger patients with bilateral nerve sparing had potency after surgery.

\section{COMMENTS}

ORP has been used as a standard technique for clinical localized prostate cancer (1-3). Since minimal invasive surgery has increased worldwide, LRP has been increasingly performed and has become a standard technique in many centers (4-6). Our hospital also uses LRP as a standard operation for clinical localized prostate cancer. A number of reported data suggest that LRP has several advantages such as small incision, fast recovery, less pain, magnified picture for accurate dissection and reduced blood loss. However, LRP is a difficult procedure that needs surgical skill and long learning curve $(7,11)$. Outcomes of radical prostatectomy are also depended on several factors including surgical skill and experience of the surgeon and surgical team. There are many reported series comparing data between ORP and LRP in the literature (4,7-9), the majority published in the western countries. Our study comparing ORP and LRP was limited to the South East Asia region. It was retrospectively carriedout to evaluate ORP and LRP in Thai men. To exclude variation of surgical skill and experience of surgeon, the first 100 cases of ORP and the first 100 cases of LRP performed by a single surgeon were compared.

Each cohort group showed the same patient characteristics except for preoperative PSA. Preoperative PSA in our series was higher than other series $(8,9,11)$. At present, there has been no official prostate cancer screening program in Thailand. In the past, most patients who had PSA testing were men with lower urinary tract symptoms (LUTS) and diagnosed as benign prostatic hyperplasia (BPH). Since there has been more prostate cancer awareness in recent years, PSA testing was used more in men without LUTS or $\mathrm{BPH}$. More patients presented with abnormal PSA regardless of LUTS or BPH. Therefore, more patients have presented with lower PSA in recent years. For these reasons, patients in ORP group who were diagnosed in the past had higher PSA than patients in LRP group who presented in recent years. However, preoperative PSA in both groups were higher than in the western countries. Another reason was that the treatment option for prostate cancer was decided by the patients, and most Thai patients preferred radical prostatectomy even when there were high risks (PSA $>10 \mathrm{ng} / \mathrm{mL}$ ).

Our data showed that LRP had significant less blood loss and blood transfusion than ORP because LRP had magnified structure of organs and surrounding tissues resulting in more accurate dissection. However, LRP had longer operative time. There was no mortality. The morbidities could be managed safely. Incontinence rate was comparable to other series (4-7). This study also concluded that LRP was a safe procedure even in the initial period.

The proportion of pathological localized disease was not different in both groups. Approximately $70 \%$ of the patients were organ confined disease and approximately $30 \%$ were locally advanced disease. For pathological organ confined disease, free margin rates in ORP and LRP were $88.9 \%$ and $91.3 \%$, respectively, which were comparable to other series $(8,9)$. This was not different between ORP and LRP. For pT3 disease, positive margin were high in both groups and not significantly different. Our data showed that surgical technique between ORP and LRP to remove cancer in terms of positive surgical margin were not different. Both ORP and LRP in our series achieved high free margin rate in organ confined disease. In terms of pathological results, we considered that our surgical technique of LRP was appropriate to be considered a standard technique used for organ confined disease. In contrast, both ORP and LRP could not achieve the accepted free margin rate in locally advanced disease. High positive margin rate in pT3 might be due to several factors such as high preoperative PSA, clinical T3 stage or surgical technique in the learn- 
ing curve period. The most common site of positive margin was apex. It requires experience to completely remove tumors that were located beyond the prostate gland with preserving enough urethral length for continence. As mention above, the proportion of pT3 in our series was almost one-third. Thus, overall positive margin rates in both groups were higher than other series. However, the overall free margin rate between ORP and LRP was not different. Since our data only concerns a limited follow-up period, the outcomes for cancer control will require long term evaluation.

All data of perioperative surgical parameters, morbidities and pathological results showed that LRP was comparable to ORP even during the period of the initial series for the surgeon. Our results suggest that LRP could be considered a standard technique as ORP for clinical localized prostate cancer in Asian men.

The procedure of radical prostatectomy is an issue that needs experiences of surgical skill and number of cases to overcome the learning curve. For ORP, the bleeding and blood transfusion rate was high. At the beginning, almost patients needed a blood transfusion. When we had more experience, bleeding and blood transfusion were reduced. For LRP, it was a very difficult procedure when we performed in the early period. Three cases of LRP that need conversion to ORP because of no progression were in the beginning period. When we had more experience, the operating time decreased from 5 hours to 2-3 hours and estimated blood loss and blood transfusion rate greatly decreased. The important factor to improve skill is to continuously perform the procedure. In Thailand, most cases of radical prostatectomy are performed at the referral center such as the university hospital. At present, ORP is increasingly performed in many hospitals. In contrast, LRP is still limited to few hospitals. Thus, most patients with clinical localized prostate cancer are referred to the university hospital. In Thailand, even the prevalence of prostate cancer is not as high as those in the western countries; more prostate cancer awareness in recent years has caused more clinical localized cancer to be detected. In addition, our university hospital is a medical center where the majority of patients with prostate cancer are referred to and therefore, we continue perform LRP on a regular basis.

\section{CONCLUSIONS}

For initial experience by a single surgeon, LRP is comparable to ORP without significant morbidity but LRP had a longer operative time. However, LRP decreased blood loss and blood transfusion. For localized prostate cancer, free surgical margin rate of ORP and LRP was not significantly different. With increasing experience the laparoscopic technique should be considered a feasible procedure for patients with prostate cancer.

\section{CONFLICT OF INTEREST}

None declared.

\section{REFERENCES}

1. Han M, Partin AW, Pound CR, Epstein JI, Walsh PC: Long-term biochemical disease-free and cancer-specific survival following anatomic radical retropubic prostatectomy. The 15-year Johns Hopkins experience. Urol Clin North Am. 2001; 28: 555-65.

2. Hull GW, Rabbani F, Abbas F, Wheeler TM, Kattan MW, Scardino PT: Cancer control with radical prostatectomy alone in 1,000 consecutive patients. J Urol. 2002; 167: 528-34.

3. Klevecka V, Burmester L, Musch M, Roggenbuck U, Kroepfl D: Intraoperative and early postoperative complications of radical retropubic prostatectomy. Urol Int. 2007; 79: 217-25.

4. Touijer K, Eastham JA, Secin FP, Romero Otero J, Serio A, Stasi J, et al.: Comprehensive prospective comparative analysis of outcomes between open and laparoscopic radical prostatectomy conducted in 2003 to 2005. J Urol. 2008; 179: 1811-7; discussion 1817.

5. Stolzenburg JU, Rabenalt R, DO M, Ho K, Dorschner W, Waldkirch E, et al.: Endoscopic extraperitoneal radical prostatectomy: oncological and functional results after 700 procedures. J Urol. 2005; 174: 1271-5; discussion 1275.

6. Goeman L, Salomon L, La De Taille A, Vordos D, Hoznek A, Yiou R, et al.: Long-term functional and oncological results after retroperitoneal laparoscopic prostatectomy according to a prospective evaluation of 550 patients. World J Urol. 2006; 24: 281-8.

7. Lepor H: Open versus laparoscopic radical prostatectomy. Rev Urol. 2005; 7: 115-27. 
8. Ghavamian R, Knoll A, Boczko J, Melman A: Comparison of operative and functional outcomes of laparoscopic radical prostatectomy and radical retropubic prostatectomy: single surgeon experience. Urology. 2006; 67: 1241-6.

9. Guazzoni G, Cestari A, Naspro R, Riva M, Centemero A, Zanoni M, et al.: Intra-and peri-operative outcomes comparing radical retropubic and laparoscopic radical prostatectomy: results from a prospective, randomised, single-surgeon study. Eur Urol. 2006; 50: 98-104.
10. Walsh PC: Anatomic radical prostatectomy: evolution of the surgical technique. J Urol. 1998; 160: 241824.

11. Ferguson GG, Ames CD, Weld KJ, Yan Y, Venkatesh R, Landman J: Prospective evaluation of learning curve for laparoscopic radical prostatectomy: identification of factors improving operative times. Urology. 2005; 66: $840-4$.
Accepted after revision: December 5, 2008

\section{Correspondence address:}

Dr. Sunai Leewansangtong

Division of Urology

Faculty of Medicine Siriraj Hospital

Mahidol University

Bangkoknoi, Bangkok 10700, Thailand

Fax: + 66 2411-2011

E-mail: sislt@mahidol.ac.th

\section{EDITORIAL COMMENT}

The paper presents an interesting retrospective study relevant to countries where daVinci robot is not widely available. In fact, it seems to be an honest report of two series of radical prostatectomies that started at different times. The author has performed open surgery for the first time in 1999 and laparoscopic procedure in 2005. After performing open radical prostatectomy for some years, he began performing it laparoscopically in elective patients. The low volume of this surgery (200 in 8 years) makes its mastery harder. Finally, the authors developed a preference for laparoscopy despite similar rates of positive margins and functional results. It would be interesting to know what the surgeon's previous experience in laparoscopy was before he started doing radical prostatectomy. Also, we could not follow the evolution of the learning curve neither in open nor in laparoscopic surgeries. The operative time was relatively short but the bleeding and the transfusion rates were higher than those in other series were. Perhaps the final message of the article is that most urologists that perform open radical prostatectomy who have had the perseverance to climb up the learning curve of radical prostatectomy eventually prefer doing it laparoscopically.
Dr. Anuar I. Mitre

Division of Urology, University of Sao Paulo, USP

Sao Paulo, SP, Brazil E-mail:anuar@mitre.com.br 


\section{EDITORIAL COMMENT}

Laparoscopic radical prostatectomy (LRP) is increasingly performed at specialized centers worldwide. With gathering experience, the laparoscopic technique has been shown to be feasible and reproducible (1).

The laparoscopic approach offers the advantages of laparoscopic surgery as less postoperative pain, fewer analgesics drugs and early mobilization. The magnification of the surgical field, allow a clear operative field with better view during the dissection of the neurovascular bundles and the urethro-vesical anastomosis.

The authors show in this paper a longer operative time with the LRP. However, LRP decreased blood loss and blood transfusion. For this population of localized prostate cancer, free surgical margin rate of ORP and LRP was not significantly different.

Outcomes of radical prostatectomy are dependent on several factors including surgical skill and experience of surgeon and surgical team. Besides, radical prostatectomy requires a sufficient number of cases to overcome the learning curve. It should be learned within an intensive teaching program (2).

\section{EDITORIAL COMMENT}

The authors present the results of a nonrandomized series within the learning curves of both radical retropubic prostatectomy and laparoscopic radical prostatectomy. The results of the laparoscopic approach are surprisingly good for the first 100 cases, even in terms of surgical time and urinary continence. This fact reflects a special skill with the laparoscopic technique acquired by the single surgeon before the beginning of this series and, at the same time, a previous huge experience with open radical prostatectomy, or a bias during the selection of the patients.

The authors should explain the extremely high rates of blood transfusion in both groups, respectively
Although long-term oncological outcomes are not available for the majority of genitourinary malignancies treated by the laparoscopic approach, the intermediate-term data are encouraging and comparable to open surgery. Multicenter studies with longer follow-up are necessary.

\section{REFERENCES}

1. Gill IS, Ukimura O, Rubinstein M, Finelli A, Moinzadeh A, Singh D, et al.: Lateral pedicle control during laparoscopic radical prostatectomy: refined technique. Urology. 2005; 65: 23-7.

2. Eden CG, Neill MG, Louie-Johnsun MW: The first 1000 cases of laparoscopic radical prostatectomy in the UK: evidence of multiple 'learning curves'. BJU Int. 2008; 19. [Epub ahead of print]

\section{Dr. Mauricio Rubinstein} Section of Urology Federal Univ. of Rio de Janeiro State Rio de Janeiro, RJ, 20270-004, Brazil E-mail:mrubins74@hotmail.com

$27 \%$ (LRP) and 55\% (ORP). These numbers are not compatible with the contemporary data from the literature.

The authors could give us their results and their position about the differences between extraperitoneal (58 cases) and transperitoneal (42 cases) laparoscopic surgery.

Dr. Lisias N. Castilho Catholic University of Campinas Campinas, SP, Brazil E-mail:lisias@dglnet.com.br 